\section{International Scientific Journal Theoretical \& Applied Science}

p-ISSN: 2308-4944 (print) $\quad$ e-ISSN: 2409-0085 (online)

Year: $2016 \quad$ Issue: 3 Volume: 35

Published: $30.03 .2016 \quad \underline{\text { http://T-Science.org }}$

SECTION 2. Applied mathematics. Mathematical modeling.
Yunona Rinatovna Krakhmaleva candidate of technical Sciences, associate Professor of Mathematics, Taraz state University named after M.Kh. Dulati, Kazakhstan yuna_kr@mail.ru

Andrey Titovets master 2-year degree in Mathematics Taraz state University named after M.Kh. Dulati, Kazakhstan

\title{
ANALYTICAL SOLUTION OF PARTIAL DIFFERENTIAL EQUATIONS WITH CONSTANT COEFFICIENTS IN THE MAPLE
}

Abstract: We consider the problem of finding analytical solutions of linear partial differential equations with constant coefficients.

Key words: Maple, analytical solution, linear equation.

Language: Russian

Citation: Krakhmaleva YR, Titovets A (2016) ANALYTICAL SOLUTION OF PARTIAL DIFFERENTIAL EQUATIONS WITH CONSTANT COEFFICIENTS IN THE MAPLE. ISJ Theoretical \& Applied Science, 03 (35): $182-185$.

Soi: http://s-o-i.org/1.1/TAS-03-35-31 Doi: crossef http://dx.doi.org/10.15863/TAS.2016.03.35.31

\section{АНАЛИТИЧЕСКОЕ РЕШЕНИЕ УРАВНЕНИЙ В ЧАСТНЫХ ПРОИЗВОДНЫХ С ПОСТОЯННЫМИ КОЭФФИЦИЕНТАМИ В СРЕДЕ МАРLЕ}

Аннотация: Рассматривается задача нахождения аналитического решения линейных уравнений 6 частных производных с постоянными коэффициентами.

Ключевые слова: Maple, аналитическое решение, линейное уравнение.

Нахождение аналитических решений уравнений в частных производных в среде Maple 17 осуществляется с помощью пакета PDEtools. Несмотря на достаточно большой набор инструментов пакета для работы с дифференциальными уравнениями в частных производных поиск их решения представляет собой достаточно сложную задачу, которая требует знания математических методов в данной области. Учитывая, тот факт, что СКМ имеют развитый язык программирования, который содержит средства процедурного программирования, имеется возможность разрабатывать универсальные алгоритмы с помощью которых можно программировать формальное построение решения уравнения в частных производных, которые будут использованы для конкретных физических задач. Рассмотрим задачу нахождения аналитического решения линейного уравнения в частных производных с постоянными коэффициентами:

$$
a_{11} \frac{\partial u}{\partial x^{2}}+2 a_{12} \frac{\partial u}{\partial x \partial y}+a_{22} \frac{\partial u}{\partial y^{2}}+a_{1} \frac{\partial u}{\partial x}+a_{2} \frac{\partial u}{\partial y}+a_{0} u+d=0
$$

где

$$
a_{11}, a_{12}, a_{22}, a_{1}, a_{2}, a_{0}, d=\text { const }
$$

$u=u(x, y) \quad$ - неизвестна функция.

Для нахождения общего решения воспользуемся стандартными средствами Maple.
Вводим значения постоянных $a_{11}, a_{12}, a_{22}, a_{1}, a_{2}, a_{0}, d$ исходного уравнения, а затем уравнение (1):

restart; with(PDEtools); 


\begin{tabular}{l|lrl|l|ll} 
& ISRA (India) & $=\mathbf{1 . 3 4 4}$ & SIS (USA) & $=\mathbf{0 . 9 1 2}$ & ICV (Poland) & $=\mathbf{6 . 6 3 0}$ \\
Impact Factor: & ISI (Dubai, UAE) $=\mathbf{0 . 8 2 9}$ & PUHU (Russia) $=\mathbf{0 . 1 7 9}$ & PIF (India) & $=\mathbf{1 . 9 4 0}$ \\
& GIF (Australia) & $\mathbf{0 . 5 6 4}$ & ESJI (KZ) & $=\mathbf{1 . 0 4 2}$ & IBI (India) & $\mathbf{4 . 2 6 0}$
\end{tabular}

$$
\begin{aligned}
& a 11:=; b:=: a 22:=; a 1:=; a 2:=; a 0:=; d:=; a 12:=\frac{b}{2} \\
& \text { PDE1 }:=a 11 \cdot \operatorname{diff}(u(x, y), x, x)+2 \cdot a 12 \cdot \operatorname{diff}(u(x, y), x, y)+a 22 \\
& \quad \cdot \operatorname{diff}(u(x, y), y, y)+a 1 \cdot \operatorname{diff}(u(x, y), x)+a 2 \cdot \operatorname{diff}(u(x, y), y)+a 0 \\
& \quad \cdot u(x, y)+d=0 ;
\end{aligned}
$$

Находим значение определителя

$$
D=a_{12}^{2}-a_{11}, a_{22},
$$

используем составной оператор условного перехода :

для последующего установления факта принадлежности к типу исходного уравнения

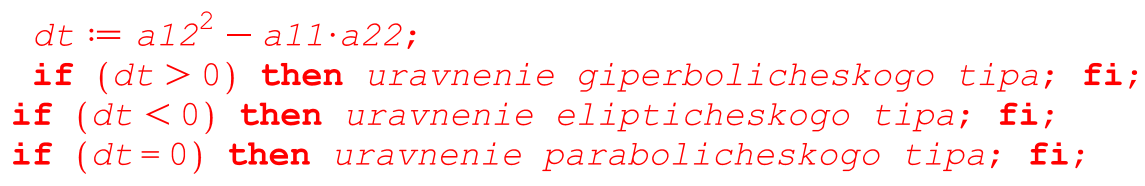

Принадлежность к типу уравнения дает возможность использовать программу Maple-

mapde ( ), которая преобразует исходное уравнение к каноническому уравнению в частных производных другого вида, которое, будет решаться командой pdsolve ( ). При этом надо иметь ввиду, программа mapde ( ) преобразовывает исходное уравнение к каноническому виду, исходя из его принадлежности к типу. Это означает, если программу записать в виде

mapde (PDE, canom ),то каноническая форма будет содержать только одну смешанную

if $(d t=0)$ then mapde(PDE1, canop); fi;

if $(d t>0)$ or $(d t<0)$ then mapde(PDE1, canom); fi; производную,что соответствует гиперболическому типу. Если же программу записать в виде

mapde (PDE, canop ),то каноническая форма будет содержать частные производные 2 -го порядка по переменным и не будет содержать смешанную производную, что соответствует эллиптическому и параболическому типу. Для того, чтобы программа работала в общем случае и сама выбирала выполнение canom или canop снова используем составной оператор условного перехода:
Общее решение находим, как указано выше, используя программу pdsolve ( ), которая найдет решение по новым переменным, согласно команды mapde ( ). Для того, чтобы записать решение в исходных переменных подключаем программy sol( ).Удостовериться в правильности нахождения найденного решения возможно с помощью команды simplify ().
Описанную программу применим для нахождения общего решения уравнения в частных производных:

$$
49 \frac{\partial u}{\partial x^{2}}-14 \frac{\partial u}{\partial x \partial y}+\frac{\partial u}{\partial y^{2}}+14 \frac{\partial u}{\partial x}-2 \frac{\partial u}{\partial y}=0
$$

restart; with(PDEtools); 


\begin{tabular}{l|lrl|l|ll} 
& ISRA (India) & $=\mathbf{1 . 3 4 4}$ & SIS (USA) & $=\mathbf{0 . 9 1 2}$ & ICV (Poland) & $=\mathbf{6 . 6 3 0}$ \\
Impact Factor: & ISI (Dubai, UAE) $=\mathbf{0 . 8 2 9}$ & PUHL (Russia) $=\mathbf{0 . 1 7 9}$ & PIF (India) & $=\mathbf{1 . 9 4 0}$ \\
& GIF (Australia) & $\mathbf{0 . 5 6 4}$ & ESJI (KZ) & $=\mathbf{1 . 0 4 2}$ & IBI (India) & $=\mathbf{4 . 2 6 0}$ \\
& JIF & $\mathbf{1 . 5 0 0}$ & SJIF (Morocco) & $=\mathbf{2 . 0 3 1}$ & & \\
\hline
\end{tabular}

$>$

$a 11:=49 ; b:=-14: a 22:=1 ; a 1:=14 ; a 2:=-2 ; a 0:=0 ; d:=-2 ; a 12:=\frac{b}{2}$;

PDE1 := a11.diff(u(x,y), $x, x)+2 \cdot a 12 \cdot \operatorname{diff}(u(x, y), x, y)+a 22$

$\cdot \operatorname{diff}(u(x, y), y, y)+a 1 \cdot \operatorname{diff}(u(x, y), x)+a 2 \cdot \operatorname{diff}(u(x, y), y)+a 0$

$\cdot u(x, y)+d=0$;

$d t:=a 12^{2}-a 11 \cdot a 22$;

if $(d t>0)$ then uravnenie giperbolicheskogo tipa; fi;

if $(d t<0)$ then uravnenie elipticheskogo tipa; fi;

if $(d t=0)$ then uravnenie parabolicheskogo tipa; fi;

if $(d t=0)$ then mapde(PDE1, canop); fi;

if $(d t>0)$ or $(d t<0)$ then mapde(PDEl,canom); fi;

$$
\begin{gathered}
a 11:=49 \\
a 22:=1 \\
a 1:=14 \\
a 2:=-2 \\
a 0:=0 \\
d:=-2 \\
a 12:=-7 \\
P D E 1:=49\left(\frac{\partial^{2}}{\partial x^{2}} u(x, y)\right)-14\left(\frac{\partial^{2}}{\partial y \partial x} u(x, y)\right)+\frac{\partial^{2}}{\partial y^{2}} u(x, y)+14\left(\frac{\partial}{\partial x} u(x, y)\right) \\
-2\left(\frac{\partial}{\partial y} u(x, y)\right)-2=0 \quad
\end{gathered}
$$$$
d t:=0
$$

uravnenieparabolicheskogo tipa

$$
\begin{aligned}
& \left(-2+14\left(\frac{\partial}{\partial \_\xi 2} u\left(\_\xi 1, \xi_{-} 2\right)\right)+49\left(\frac{\partial^{2}}{\partial \_\xi 2^{2}} u\left(\_\xi 1, \xi_{-} 2\right)\right)\right) \& w h e r e\left\{\left\{\xi 1=y+\frac{1}{7} x, \xi_{2}\right.\right. \\
& =x\}
\end{aligned}
$$

$>$

$>o p(\%)$

$$
-2+14\left(\frac{\partial}{\partial \_\xi 2} u\left(\_\xi 1, \xi_{-} 2\right)\right)+49\left(\frac{\partial^{2}}{\partial \_\xi 2^{2}} u\left(\_\xi 1, \_\xi 2\right)\right),\left\{-\xi 1=y+\frac{1}{7} x, \_\xi 2=x\right\}
$$

$>$ pdsolve( $\left(\frac{0}{\circ}[1]\right)$;

$$
u\left(\xi \xi 1, \_\xi 2\right)=-\frac{7}{2} \mathrm{e}^{-\frac{2}{7}-\xi 2}-F 1\left(\xi_{1}\right)+\frac{1}{7}-\xi 2+\_F 2\left(\_\xi 1\right)
$$

$>\operatorname{sol}:=u(x, y)=\operatorname{subs}(\% \%[2], \operatorname{rhs}(\%))$;

$$
\text { sol }:=u(x, y)=-\frac{7}{2} \mathrm{e}^{-\frac{2}{7} x} F 1\left(y+\frac{1}{7} x\right)+\frac{1}{7} x+{ }_{-} F 2\left(y+\frac{1}{7} x\right)
$$

$>$ simplify(subs(sol, PDEI)); 


\begin{tabular}{l|lrl|l|ll} 
& ISRA (India) & $=\mathbf{1 . 3 4 4}$ & SIS (USA) & $=\mathbf{0 . 9 1 2}$ & ICV (Poland) & $=\mathbf{6 . 6 3 0}$ \\
Impact Factor: & ISI (Dubai, UAE) $=\mathbf{0 . 8 2 9}$ & PUHL (Russia) $=\mathbf{0 . 1 7 9}$ & PIF (India) & $=\mathbf{1 . 9 4 0}$ \\
& GIF (Australia) & $\mathbf{0 . 5 6 4}$ & ESJI (KZ) & $=\mathbf{1 . 0 4 2}$ & IBI (India) & $=\mathbf{4 . 2 6 0}$ \\
& JIF & $\mathbf{1 . 5 0 0}$ & SJIF (Morocco) & $=\mathbf{2 . 0 3 1}$ & & \\
\hline
\end{tabular}

$$
\begin{aligned}
& 49\left(\frac{\partial^{2}}{\partial x^{2}}\left(-\frac{7}{2} \mathrm{e}^{-\frac{2}{7} x} F 1\left(y+\frac{1}{7} x\right)+\frac{1}{7} x+{ }_{-} F 2\left(y+\frac{1}{7} x\right)\right)\right)-14\left(\frac{\partial^{2}}{\partial y \partial x}(\right. \\
& \left.\left.\quad-\frac{7}{2} \mathrm{e}^{-\frac{2}{7} x} F 1\left(y+\frac{1}{7} x\right)+\frac{1}{7} x+{ }_{-} F 2\left(y+\frac{1}{7} x\right)\right)\right)+\frac{\partial^{2}}{\partial y^{2}}\left(-\frac{7}{2} \mathrm{e}^{-\frac{2}{7} x} F 1(y\right. \\
& \left.\left.\quad+\frac{1}{7} x\right)+\frac{1}{7} x+{ }_{-} F 2\left(y+\frac{1}{7} x\right)\right)+14\left(\frac { \partial } { \partial x } \left(-\frac{7}{2} \mathrm{e}^{-\frac{2}{7} x} F 1\left(y+\frac{1}{7} x\right)+\frac{1}{7} x\right.\right. \\
& \left.\left.\quad+{ }_{-} F 2\left(y+\frac{1}{7} x\right)\right)\right)-2\left(\frac { \partial } { \partial y } \left(-\frac{7}{2} \mathrm{e}_{-}^{-\frac{2}{7} x} F 1\left(y+\frac{1}{7} x\right)+\frac{1}{7} x+{ }_{F} F 2(y\right.\right. \\
& \left.\left.\left.\quad+\frac{1}{7} x\right)\right)\right)-2=0
\end{aligned}
$$

simplify $\left(\right.$ Ihs $\left.\left(\frac{\circ}{0}\right)\right)$;

0

Таким образом,

$$
u(x, y)=-\frac{7}{2} e^{\frac{2}{7}{ }^{4}} F_{1}\left(y+\frac{1}{7} x\right)+F_{2}\left(y+\frac{1}{7} x\right)+\frac{1}{7} x
$$

$F_{1}, F_{2}$ - постоянные.

\section{References:}

1. Bitsadze AV (1982) Uravneniya matematicheskoy fiziki. Moscow: Nauka, 1982, $-336 \mathrm{p}$.

2. Vladimirov VS (1981) Uravneniya matematicheskoy fiziki. Moscow: Nauka, 1981, $-512 \mathrm{p}$.

3. Mikhaylov VP (1983) Differentsial'nye uravneniya $\mathrm{S}$ chastnymi proizvodnymi. Moscow: Nauka, 1983, -424p.

4. Goloskokov DP (2004) Uravneniya matematicheskoy fiziki. Reshenie zadach v sisteme Maple uchebnik dlya vuzov - SPb.: Piter, 2004.-539 p.

5. D'yakonov VP (2006) Maple 9.5/10 v matematike, fizike i obrazovanii Izd: Piter, 2006.

6. (2010) «Reshenie obyknovennykh differentsial'nykh uravneniy v pakete Maple», Metodicheskie ukazaniya $\mathrm{k}$ provedeniyu prakticheskikh zanyatiy. Novokuznetsk 2010.

7. (2012) «Postroenie grafikov v pakete Maple», Novokuznetsk 2012.
8. Sdvizhkov OA (2003) «Matematika na komp'yutere: Maple 8», Moscow SOLON PRESS 2003.

9. (2016) Kratkoe posobie po Maple. 2016. Available: http://mif.vspu.ru/books/mapletut/page4.html (Accessed: 20.03.2016). 